\title{
Influence of prostate weight, obesity and height on surgical outcomes of robot-assisted laparoscopic radical prostatectomy in Korean men
}

\author{
Minji Jennifer Kim · Sung Yul Park · Koon Ho Rha
}

Received: 29 August 2007 / Accepted: 5 December 2007 / Published online: 21 December 2007

(C) Springer London 2007

\begin{abstract}
The purpose of this study was to determine if different body habitus, including prostate weight, body mass index (BMI) and height, are associated with surgical outcomes in patients undergoing robot-assisted laparoscopic radical prostatectomy (RLRP). Between July 2005 and June 2007, 135 patients underwent RLRP by a single surgeon. Information was collected retrospectively on prostate weight, BMI and height. Surgical outcome was assessed by total operative time, estimated blood loss (EBL) and surgical margin as parameters of technical difficulty. When statistically analyzed, prostate size was significantly related to total operative time $(P=0.001)$ and $\mathrm{EBL}$ $(P=0.033)$. But prostate size was not significantly related to the surgical margin. Patient BMI and height did not appear to affect surgical outcomes. Despite a shift in body frames towards the western standard, most Asians still have shorter stature, lower BMI and smaller prostates. We have found from our experience that different body habitus is not significantly related to surgical outcomes of RLRP, and therefore, it seems promising to operate successfully on Asian patients. However, it is important to obtain long-term outcomes on a larger cohort of patients in order to confirm our findings.
\end{abstract}

Keywords Robotics · Prostatectomy · Obesity

M. J. Kim · S. Y. Park · K. H. Rha ( $ه)$

Department of Urology, Urological Science Institute and Brain Korea 21 Project for Medical Science,

Yonsei University College of Medicine,

134, Shinchon-dong, Seodaemun-gu,

Seoul 120-752, South Korea

e-mail:khrha@yuhs.ac

\section{Introduction}

Recent medical advances have made a significant change of scene in operating rooms. Laparoscopic radical prostatectomy (LRP), which was introduced only just over a decade ago, was thought to be an effective method of controlling prostate cancer, but it still had technical difficulties [1]. Surgeon's 2D vision and rigid instrumental movements limited the surgical field, especially in patients with physical variation [2]. Now robot-assisted laparoscopic radical prostatectomy (RLRP) is an emerging surgical option for patients with prostate cancer.

RLRP is an innovative version of LRP. Since the introduction of RLRP a few years ago, a number of studies have shown that its minimally invasive approach has overcome the technical challenges faced by LRP, although less is known about its patient feasibility [3, 4]. Advantages of RLRP became especially valuable in more complicated cases such as operating on obese patients [5-7].

Recent reports claim that the technical difficulties resulting from physical variation have been overcome, but at this stage, it remains controversial. Most Asians have shorter stature, lower BMI and smaller prostates [8, 9]. These differences from the western population may lead to varied surgical outcomes of RLRP. The objective of this study is to assess the hypothesis that the size of the prostate, obesity and height influence surgical outcomes. Our research focuses on Korean patients undergoing RLRP, and we hope this study will contribute towards the expanding field of RLRP among the Asian population.

\section{Materials and methods}

Between July 2005 and June 2007, 135 patients underwent RLRP by a single surgeon. This was a non-randomized, 
retrospective study evaluating all of our RLRP patients, and we did not limit surgical candidacy by previous surgical history or body habitus. Information was recorded using patient charts retrospectively on prostate weight, body mass index (BMI) and height. Technical difficulties and overall surgical outcomes were assessed based on total operative time, estimated blood loss (EBL) and surgical margin status. In all cases, at least two of three data sources were reviewed.

Patients were divided into three groups according to their pathologic prostate weight. Patients were classified as having a small prostate if it weighed less than $30 \mathrm{~g}$ (group 1), normal between 30 and $50 \mathrm{~g}$ (group 2) and large if greater than $50 \mathrm{~g}$ (group 3).

The World Health Organization describes BMI as a simple index of weight for height that is commonly used to classify underweight, overweight and obese adults. It is defined as the weight in kilograms divided by the square of the height in meters $\left(\mathrm{kg} / \mathrm{m}^{2}\right)$. However, the values are based on western populations, and it may not be exactly suitable to use in Asians. Therefore, we used a different BMI scale specifically designed to be applicable to Asian males that was announced recently. The current WHO Classification of BMI states that patients over $25 \mathrm{~kg} / \mathrm{m}$ are considered overweight [8]. In our study, we used $23 \mathrm{~kg} / \mathrm{m}^{2}$ instead as the cutoff point, which was introduced by WHO experts. Patients were considered to be of normal weight with a BMI greater than $23 \mathrm{~kg} / \mathrm{m}^{2}$ (group 1), overweight with greater than $23 \mathrm{~kg} / \mathrm{m}^{2}$ but less than $27.5 \mathrm{~kg} / \mathrm{m}^{2}$ (group 2), and obese with $27.5 \mathrm{~kg} / \mathrm{m}^{2}$ or greater (group 3).

Individuals were considered to be of short stature (group 1) if their height was equal to or less than the first lower quartile of the height distribution of the population of the same gender. Individuals whose heights fell in the second and third quartiles were classified as being of normal or average stature (group 2). Individuals above the third quartile were classified as tall (group 3) [10]. Statistical values of height were obtained from the Korea National Statistical Office. The average height of Korean males above the age of 60 was $161 \mathrm{~cm}$ [11]. Patients below $161 \mathrm{~cm}$ were classified as being short (group 1), between 161 and $168 \mathrm{~cm}$ as being average height (group 2), and tall (group 3) above $168 \mathrm{~cm}$.

Surgical outcome was assessed by total operative time, EBL and surgical margin as parameters of technical difficulty. Total operative time was defined as the time between the start of Veress needle insertion and skin closure including the time spent for robot preparation and docking [12]. The attending anesthesiologist recorded EBL during surgery based on the amount of fluid collected by the suction device. Surgical margin status was divided into positive or negative.

RLRP was performed using the da Vinci Robotic System $^{\mathrm{TM}}$ (Intuitive Surgical, Mountain View, CA). Our surgical technique was based on Vattikuti Institute Prostatectomy with slight modification [13].

Statistical evaluation was made using SPSS, and comparative analysis was done using Spearman correlation coefficient and ANOVA, with $P<0.05$ considered as statistically significant.

\section{Results}

The mean age and preoperative prostate-specific antigen (PSA) of 135 patients undergoing RLRP were 64 and $8.0 \mathrm{ng} / \mathrm{ml}$, respectively. The mean BMI, prostate weight and height of 135 patients were $24.3 \mathrm{~kg} / \mathrm{m}^{2}, 5.5 \mathrm{~g}$ and $169 \mathrm{~cm}$, respectively. The mean operative time was $220 \mathrm{~min}$ (range 149-418). Total operative time was significantly related to prostate weight $(P=0.001)$. It appeared from statistical analysis that heavier prostate weight was significantly related to longer operative time. BMI and height did not significantly affect total operative time. The mean EBL was $413 \mathrm{ml}$ (range 50-2,700). The EBL was not significantly related to BMI or height. When compared within different prostate weight groups, EBL was not significantly related to prostate weight $(P=0.329)$. However, a significant positive relationship was found between prostate weight and EBL when different parameters were compared $(P=0.033)$. The positive margin rate was present in 41 cases out of a total of 135 patients. Margin status was not significantly related to surgical outcomes either within groups or between parameters. The relationship between surgical outcomes and each variable is shown in Table 1.

\section{Discussion}

Soon after Schuessler et al. [1] first attempted LRP in 1992, massive worldwide interest in minimally invasive surgery was initiated, and many drawbacks of open prostatectomy were overcome [14]. However, reduced tactile feedback and inability of the surgeon to control the view of the surgical fields and two-dimensional vision remained as potential disadvantages of LRP $[2,15]$.

Extensive studies have been done to investigate relationship between patient body habitus and perioperative complications in LRP patients. This has been reported by other LRP series using different techniques [16-19]. Chang et al. [20] reported significantly longer operative times for 400 patients undergoing LRP within larger prostate weight groups. However, Boczko et al. [21] described that the only significant difference in operative parameters of RLRP was a small increase in blood loss in patients with large prostate size. No impact was seen on total positive margin status 
Table 1 Surgical outcomes of RLRP according to different body habitus

\begin{tabular}{|c|c|c|c|c|c|c|c|c|c|c|c|c|}
\hline & \multicolumn{4}{|c|}{ Prostate weight groups ${ }^{\mathrm{a}}$} & \multicolumn{4}{|c|}{ BMI groups ${ }^{b}$} & \multicolumn{4}{|c|}{ Height groups ${ }^{c}$} \\
\hline & Group 1 & Group 2 & Group 3 & $P$ value & Group 1 & Group 2 & Group 3 & $P$ value & Group 1 & Group 2 & Group 3 & $P$ value \\
\hline OT (min) & 215 & 222 & 253 & 0.043 & 217 & 221 & 227 & 0.825 & 241 & 228 & 212 & 0.069 \\
\hline $\mathrm{EBL}(\mathrm{ml})$ & 398 & 389 & 604 & 0.329 & 424 & 410 & 415 & 0.984 & 440 & 483 & 377 & 0.382 \\
\hline SM (\%) & 27.1 & 41.7 & 23.1 & 0.569 & 23.7 & 31.3 & 38.5 & 0.268 & 37.5 & 30.6 & 28.9 & 0.53 \\
\hline
\end{tabular}

$R L R P$ robot-assisted laparoscopic radical prostatectomy, $O T$ opertative time, $E B L$ estimated blood loss, SM surgical margin

${ }^{a}$ Prostate weight groups 1, small (under $30 \mathrm{~g}$ ); 2, normal (between 30-50 g); 3, large (over $50 \mathrm{~g}$ )

b BMI groups 1, normal (under $23 \mathrm{~kg} / \mathrm{m}$ ); 2, overweight (23-27.5 kg/m); 3, obese (over $27.4 \mathrm{~kg} / \mathrm{m}$ )

${ }^{\mathrm{c}}$ Height groups 1, short (under $\left.161 \mathrm{~cm}\right)$; 2, normal (161-168 cm); 3, tall (over $168 \mathrm{~cm}$ )

and operative times. In our experience, we have found that the operative parameters affected by increasing prostate size were operative times and EBL. Surgical margin status was not influenced by varying prostate size.

LRP is no different from other operations in that thin patients are normally easier to operate on compared to obese patients [17, 22]. Whether or not this has an effect on perioperative outcomes is not clear.

Not long after the development of LRP, Binder and Kramer [23] performed the first RLRP at Frankfurt University using the Da Vinci System ${ }^{\mathrm{TM}}$ in May 2000. RLRP is more favorable compared to LRP in that it has a more gradual learning curve. Technical factors of RLRP that may direct towards a favorable position include 3D vision of the surgical field, increased degrees of free movement and filtered hand tremor. While RLRP appears to be an effective surgical option for prostatectomy, lack of dexterity remains a major disadvantage. Other obstacles to be overcome include its high cost and the bulky machinery itself $[2,4$, $15,24]$. With regard to RLRP and patient outcomes, the current data available are not completely mature. Nevertheless, it has been suggested that body habitus may lead to a less favorable outcome. Hemal et al. [25] emphasize that subtle adjustments to standard port placement are required for patient consideration and optimal surgical results. Ahlering et al. [26] reported on their results comparing patients undergoing RLRP classified by their BMI (over $30 \mathrm{~kg} / \mathrm{m}$ as obese). Greater blood loss and longer operative time were noted in 19 obese patients than in 81 who were not obese. Obese patients may require longer instruments. However, in a recent study done by Zorn et al. [19] differences in prostate weight did not affect surgical outcomes of RLRP significantly.

Despite its unknown feasibility, RLRP has been adapted by medical centers worldwide since its first trial. In Korea, the first RLRP was performed and documented by Lee et al. [27]. Prostate cancer is a relatively less common type of cancer in Korea compared to western countries. Among men over the age of 65 years, it is the fifth most common cancer. However, despite its low rates, a recent study done by Park et al. [28] showed a trend of increasing incidences of prostate cancer in most parts of Korea. Although no clear explanation has been made, factors including fast westernization and change in diet, such as increased meat and fat intake, are linked to elevated prostate cancer risk. It is therefore crucial to have a structured understanding of the disease and its effective treatment specific to Koreans.

Although we have not encountered any major complications due to different body habitus among patients, it must be emphasized that the present technique of RLRP is designed to suit western physiques.

\section{Conclusion}

A review of the published MEDLINE literature is currently insufficient to determine the surgical feasibility of RLRP in the Asian population. Despite a shift in body frame towards western standards, most Asians still have shorter stature, lower BMI and smaller prostates. We have found from our experience that different body habitus is not significantly related to surgical outcomes of RLRP, and therefore it seems promising to operate successfully on the Asian population. However, if the trend of westernization is to be continued, different consequences may follow. It is therefore important to obtain long-term outcomes on a larger cohort of patients in order to confirm our findings. Furthermore, it must be emphasized that the data used in this study are nonrandomized and intra-institutional. Caution must be taken when interpreting the results and understanding their limits.

\section{References}

1. Schuessler WW, Schulam PG, Clayman RV et al (1997) Laparoscopic radical prostatectomy: initial short-term experience. Urology 50:854-857

2. Rozet F, Harmon J, Cathelineau X et al (2006) Robot-assisted versus pure laparoscopic radical prostatectomy. World J Urol 24:171-179

3. Hu JC, Nelson RA, Wilson TG et al (2006) Perioperative complications of laparoscopic and robotic assisted laparoscopic radical prostatectomy. J Urol 175:541-546; discussion 6 
4. Goldstraw MA, Patil K, Anderson C, et al. (2007) A selected review and personal experience with robotic prostatectomy: implications for adoption of this new technology in the United Kingdom. Prostate Cancer Prostatic Dis. doi:10.1038/sj.pcan.4500968

5. Mikhail AA, Stockton BR, Orvieto MA et al (2006) Roboticassisted laparoscopic prostatectomy in overweight and obese patients. Urology 67:774-779

6. Khaira HS, Bruyere F, O'Malley PJ et al (2006) Does obesity influence the operative course or complications of robot-assisted laparoscopic prostatectomy. BJU Int 98:1275-1278; discussion 8

7. Farnham SB, Webster TM, Herrell SD et al (2006) Intraoperative blood loss and transfusion requirements for robotic-assisted radical prostatectomy versus radical retropubic prostatectomy. Urology 67:360-363

8. WHO Expert Consultation (2004) Appropriate body-mass index for Asian populations and its implications for policy and intervention strategies. Lancet 10:363:157-63

9. Jin B, Turner L, Zhou Z et al (1999) Ethnicity and migration as determinants of human prostate size. J Clin Endocrinol Metab 84:3613-3619

10. Florencio TT, Ferreira HS, Cavalcante JC et al (2007) Short stature, abdominal obesity, insulin resistance and alterations in lipid profile in very low-income women living in Maceio, north-eastern Brazil. Eur J Cardiovasc Prev Rehabil 14:346-348

11. Size Korea (2005) Korean Agency for Technology and Standards

12. Hsu EI, Hong EK, Lepor H (2003) Influence of body weight and prostate volume on intraoperative, perioperative, and postoperative outcomes after radical retropubic prostatectomy. Urology 61:601-606

13. Menon M, Shrivastava A, Kaul S et al (2007) Vattikuti Institute prostatectomy: contemporary technique and analysis of results. Eur Urol 51:648-57; discussion 57-58

14. Tewari A, Peabody J, Sarle R et al (2002) Technique of da Vinci robot-assisted anatomic radical prostatectomy. Urology 60:569572

15. Herrmann TR, Rabenalt R, Stolzenburg JU et al (2007) Oncological and functional results of open, robot-assisted and laparoscopic radical prostatectomy: does surgical approach and surgical experience matter? World J Urol 25:149-160
16. Chang SS, Duong DT, Wells N et al (2004) Predicting blood loss and transfusion requirements during radical prostatectomy: the significant negative impact of increasing body mass index. J Urol 171:1861-1865

17. El-Feel A, Davis JW, Deger S et al (2003) Laparoscopic radical prostatectomy-an analysis of factors affecting operating time. Urology 62:314-318

18. Sarle R, Tewari A, Hemal AK et al (2005) Robotic-assisted anatomic radical prostatectomy: technical difficulties due to a large median lobe. Urol Int 74:92-94

19. Zorn KC, Orvieto MA, Mikhail AA et al (2007) Effect of prostate weight on operative and postoperative outcomes of robotic-assisted laparoscopic prostatectomy. Urology 69:300-305

20. Chang CM, Moon D, Gianduzzo TR et al (2005) The impact of prostate size in laparoscopic radical prostatectomy. Eur Urol 48:285-290

21. Boczko J, Erturk E, Golijanin D et al (2007) Impact of prostate size in robot-assisted radical prostatectomy. J Endourol 21:184 188

22. Chang IH, Byun SS, Hong SK et al (2007) Assessing the body mass index of patients might help to predict blood loss during radical retropubic prostatectomy in Korean men. BJU Int 99:570-574

23. Binder J, Kramer W (2001) Robotically-assisted laparoscopic radical prostatectomy. BJU Int 87:408-410

24. Atug F, Castle EP, Srivastav SK et al (2006) Positive surgical margins in robotic-assisted radical prostatectomy: impact of learning curve on oncologic outcomes. Eur Urol 49:866-871; discussion $71-72$

25. Hemal AK, Eun D, Tewari A et al (2004) Nuances in the optimum placement of ports in pelvic and upper urinary tract surgery using the da Vinci robot. Urol Clin North Am 31:683-692, viii

26. Ahlering TE, Eichel L, Edwards R et al (2005) Impact of obesity on clinical outcomes in robotic prostatectomy. Urology 65:740 744

27. Lee YS, Han WK, Oh YT et al (2007) Robot-assisted laparoscopic radical prostatectomy: four cases. Yonsei Med J 48:341-346

28. Park SK, Sakoda LC, Kang D et al (2006) Rising prostate cancer rates in South Korea. Prostate 66:1285-1291 